\title{
Could an isolated human body lower limb model predict leg biomechanical response of Chinese pedestrians in vehicle collisions?
}

\author{
HUAXING MA ${ }^{1}$, ZHENGYU MAO $^{1}$, GUIBING Li ${ }^{1 *}$, LINGBO YAN ${ }^{2,3}$, FUHAO MO ${ }^{2}$ \\ ${ }^{1}$ School of Mechanical Engineering, Hunan University of Science and Technology, Xiangtan, China. \\ ${ }^{2}$ State Key Laboratory of Advanced Design and Manufacturing for Vehicle Body, Hunan University, Changsha, China. \\ ${ }^{3}$ Hunan SAF Automobile Technology Co., Ltd., Changsha, China.
}

\begin{abstract}
Purpose: The purpose of the current study was to investigate whether an isolated human body lower limb FE model could predict leg kinematics and biomechanical response of a full body Chinese pedestrian model in vehicle collisions. Methods: A human body lower limb FE model representing midsize Chinese adult male anthropometry was employed with different upper body weight attachments being evaluated by comparing the predictions to those of a full body pedestrian model in vehicle-to-pedestrian collisions considering different front-end shapes. Results: The results indicate that upper body mass has a significant influence on pedestrian lower limb injury risk, the effect varies from vehicle front-end shape and is more remarkable to the femur and knee ligaments than to the tibia. In particular, the upper body mass can generally increase femur and knee ligaments injury risk, but has no obvious effect on the injury risk of tibia. The results also show that a higher attached buttock mass is needed for isolated pedestrian lower limb model for impacts with vehicles of higher bonnet leading edge. Conclusions: The findings of this study may suggest that it is necessary to consider vehicle shape variation in assessment of vehicle pedestrian protection performance and leg-form impactors with adaptive upper body mass should be used for vehicles with different front-end shapes, and the use of regional leg-form impactor modeling the local anthropometry to evaluate the actual lower limb injury of pedestrians in different countries and regions.
\end{abstract}

Key words: isolated lower limb model, pedestrian leg injury, vehicle-to-pedestrian collision

\section{Introduction}

The WHO report indicates that traffic accidents caused about 1.35 million fatalities in 2016, more than $20 \%$ of which were pedestrians [29]. Real world collision data show that lower limb injuries account for more than $30 \%$ of all pedestrian AIS2+ (Abbreviated Injury Scale level 2-6) injuries [12], [22], and current vehicles still post high threat to pedestrians, even though great progress has been made in improving vehicle front-end design [15], [23]. Moreover, as a developing country with typical mixed traffic and the world's largest car market, China has a significantly higher pedestrian accident rate than other developed coun- tries. Meanwhile, pedestrian safety has not been considered in the China New Car Assessment Program (C-NCAP) until 2018 [4], the safety situation of vehicle fleets in Chinese market might be much worse than developed countries. Thus, safer vehicle frontend design for pedestrian lower limb protection is still an urgent pursuit in China.

Pedestrian safety regulations, such as the New Car Assessment Programs (NCAPs) in different countries and regions, have shown significant effect on forcing improvement of vehicle front-end design for pedestrian protection. In pedestrian safety regulations, the subsystem test procedure using an isolated leg-form impactor (such as the Flex-PLI leg-form) is employed for evaluation of vehicle safety performance (C-

\footnotetext{
* Corresponding author: Guibing Li, \#A413 Ligong Building, Hunan University of Science and Technology, Taoyuan Road, 411201 Xiangtan, China. E-mail: li8747@hotmail.com

Received: April 17th, 2020

Accepted for publication: July 1st, 2020
} 
NCAP, 2018; Euro-NCAP, 2017; J-NCAP, 2014) [4], [5], [8]. However, the isolated leg-form impactor used in current NCAPs was developed based on the anthropometry of Westerners midsize adult male [9], [10], which may not represent the human body damage situation of Chinese given the big gap in lower limb anthropometry between Chinese and Westerners (e.g., knee height of 50th percentile Chinese adult male is $442 \mathrm{~mm}$, while the data for U.S. is $493 \mathrm{~mm}$ ) [6] and the significant influence of relative height of knee versus bumper on pedestrian lower limb injury risk observed from real world crashes [12]. This may suggest that the effectiveness of C-NCAP on forcing improvement of vehicle front-end design for pedestrian lower limb protection might be undoubted for Westerners, however it may not be equally effective for Chinese. Therefore, pedestrian safety regulation of China is likely to be more effective if using an isolated leg-form impactor which can represent Chinese anthropometry in the subsystem test procedure. However, it is still lack of understanding on what kind of an isolated lower limb could represent the leg biomechanical response of Chinese pedestrians in vehicleto-pedestrian collisions, especially considering the fact that China does not have such a lower limb physical leg-form impactor like Flex-PLI.

With the help of computer technology, numerical human body models provide an important tool for vehicle safety analysis with the low cost, good repeatability and high biofidelity, where human body finite element (FE) models are widely used in pedestrian safety studies [14], [17], [25], [26]. However, most of the existing human body lower limb FE models are also based on the anthropometry of Westerners [1], [21], [27]. Mo et al. [18] developed a human body lower limb FE model of midsize Chinese adult male, but an isolation study to this model is still needed for its predictions of leg response in vehicle-to-pedestrian collisions, which could provide useful suggestions for the development of physical leg-form impactors suiting China conditions in the near future.

Therefore, the purpose of the current study was to investigate whether an isolated human body lower limb FE model could predict leg kinematics and biomechanical response in vehicle collisions considering different vehicle front-end shapes. To achieve this goal, a human body lower limb FE model representing midsize Chinese adult male anthropometry was employed with different upper body weight attachments being evaluated by comparing the predictions to those of a full body pedestrian model in vehicle-to-pedestrian collisions considering low and high bumper and bonnet leading edge designs.

\section{Materials and methods}

\subsection{Isolated human body lower limb FE model}

The human body lower limb model with detailed anatomy, called Human Active Lower Limb Model (HALLM), was used in the current work to predict the risk of pedestrian lower limb injury in a vehicle collision. The FE model of the human lower extremity model, in which bone and soft tissue are modeled in detail is shown in Fig. 1. This finite element model of the lower extremity was originally developed by researchers at Hunan University, based on CT and MRI scan data of a Chinese male (height $=1713 \mathrm{~mm}$, weight $=69.7 \mathrm{~kg}$ ), with its size is close to that of a 50th percentile Chinese adult male (AM). Then a slight scaling was carried out to make the model meeting the anthropometry of 50th percentile Chinese adult male [18]. The original lower extremity model was later configured as a standing position and verified by a corpse test of four-point bending of the knee and lateral bending and shearing of the leg [6]. In the validation study, the current prediction of the human lower extremity model showed a good match with the cadaver test data [6]. The difference in anthropometry between the HALLM and 50th percentile U.S. adult male was also shown in Fig. 1, where big gap can be clearly ob-

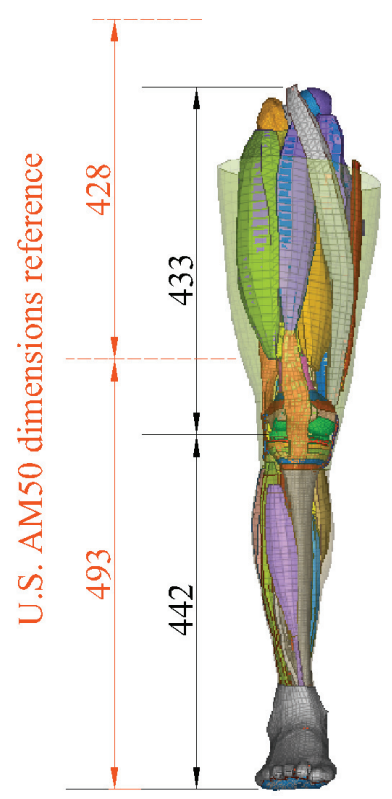

(a)

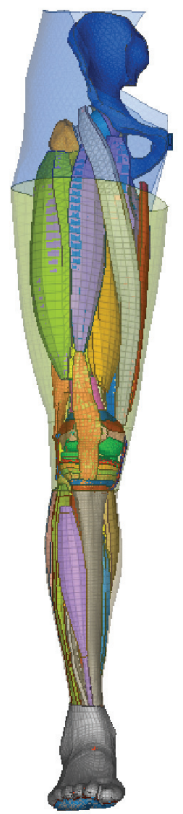

(b)
Fig. 1. FE models of the human body lower limb without buttock (a) and attached buttock (b) 
served, especially for the knee height (HALLM $442 \mathrm{~mm}$ vs. 50th percentile U.S. adult male $493 \mathrm{~mm}$ ). The HALLM model has been used to analyze the injury of the driver lower limbs in front of the vehicle [18], active muscle control [19], foot tissue injury [20] and pedestrian lower limb injury [13].

To find an isolated human body lower limb FE model which can predict leg kinematics and biomechanical response of Chinese pedestrians in vehicle collisions, isolated lower limb models without buttock (Fig. 1a) and with attached buttock (Fig. 1b) of different mass (from 1 to $7 \mathrm{~kg}$ ) were employed. The selection of these isolated models was based on previous studies of development of impactors for vehicle safety performance [3], [11].

\subsection{Vehicle front-end buck FE models}

A simplified vehicle front-end buck FE model, named as Shape 1, was defined based on the frame structure of the car's midline of a sedan, which has been used in previous pedestrian lower limb injury studies [13], [16], (Fig. 2a). Then a vehicle front-end buck FE model (Shape 2) was scaled from the Shape 1 model to consider a higher bonnet leading edge and bumper, since the height of these parts may have significant influence on the effect of upper body mass for lower limb injury risk [3]. The vehicle front-end buck models include the bonnet leading edge (BLE), bumper beam, bumper absorber and lower bumper stiffener (LBS) structures. All of the frames in the buck model are modeled by shell elements, while the lower bumper stiffeners are simulated using solid elements. The material of the frame is defined as the corresponding part in the initial model, but the thickness is changed to maintain the equivalent front-end stiffness [28]. This

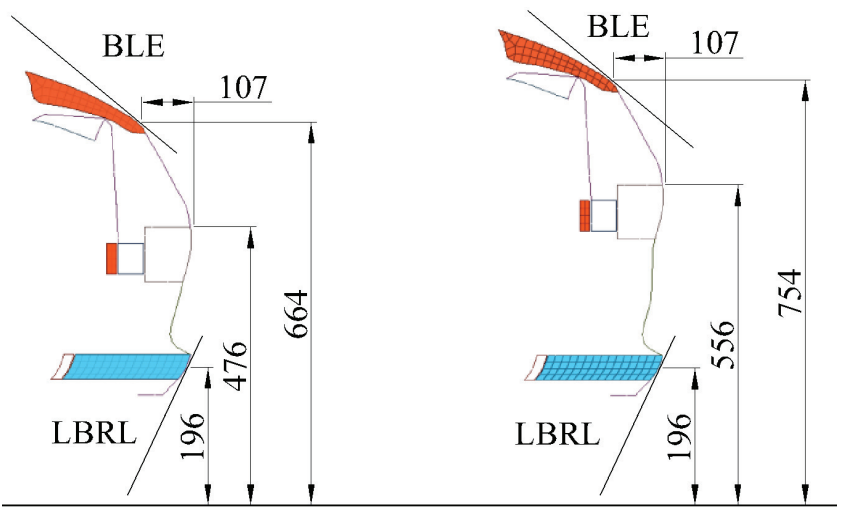

(a) (b) simplified approach has been widely adopted in previous studies based on physical shock testing and/or numerical simulation of pedestrian safety [28], [30]. The selected vehicle models generally represent the most popular vehicles in Chinese market.

\subsection{Vehicle-to-pedestrian impact simulations}

Vehicle-to-pedestrian impact simulations with the vehicle front-end buck models (Section 2.2) striking different isolated lower limb models (defined in Section 2.1) and a full body human body model were defined (Fig. 3). The full body model (Fig. 3b) formed by coupling the Chinese human body lower limb model and the upper body model of the 50th percentile Chinese dummy model [7] was developed as the benchmark to evaluate the prediction capability of different isolated lower limb models. This coupling approach has been widely used in previous study of human lower limb injury [2], [17], [18], [25]. Similarly to current pedestrian safety regulations [4], [5], in the impact simulations for isolated models an initial impact speed at $40 \mathrm{~km} / \mathrm{h}$ was defined to the isolated lower limb model and the vehicle front-end buck model was fixed fully (Fig. 3a). The knee was set at the straight posture to consider the gait stance with a high injury risk to pedestrian lower limb [16]. For the full body impact simulation, an initial impact speed of

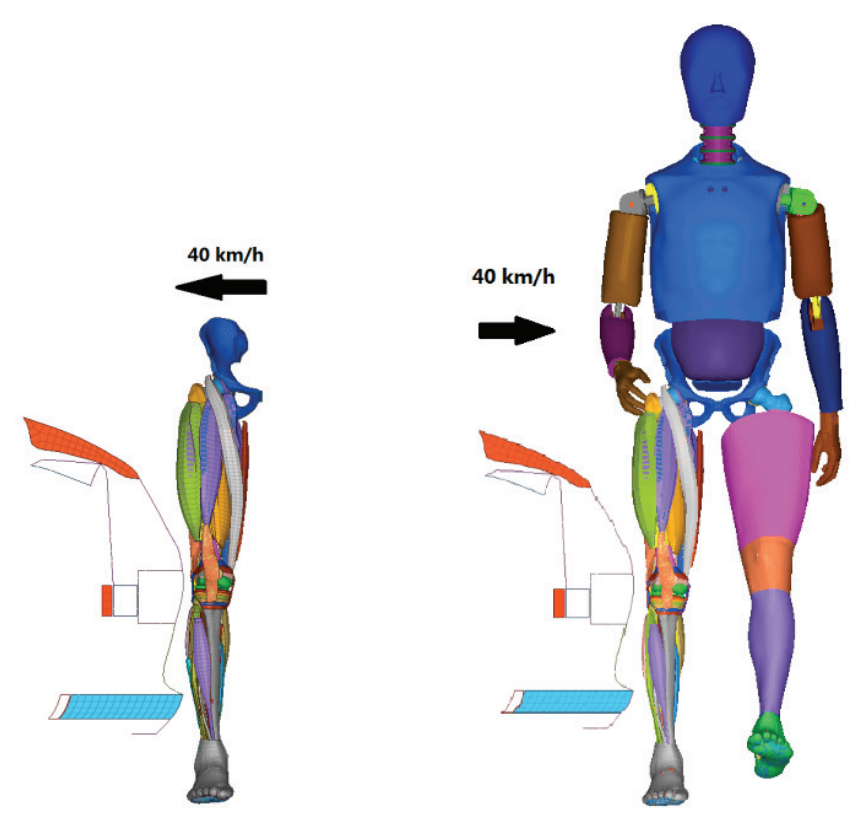

(a)

(b)

Fig. 2. Vehicle front-end buck models: (a) Shape 1 and (b) Shape 2

Fig. 3. Vehicle-to-pedestrian impact models: (a) Isolated impacts and (b) Full impacts 
$40 \mathrm{~km} / \mathrm{h}$ was defined to the vehicle model, the struck leg of the human body model was set straightly standing on the ground with a forwarding non-struck leg to simulate a walking gait stance (Fig. 3b).

\subsection{Injury risk assessment metrics}

Long bone lateral bending moment and knee ligament global strain are used as the key predictors of pedestrian lower limb injury risk in current pedestrian safety regulations [4], [5]. For the femur, lateral bending moments at the section of proximal (Femurup), mid-shaft (Femur-mid) and distal (Femur-low) level were used to assess injury risk (Fig. 4). Similarly, bending moment measurements to the tibia were also considered, which are defied as Tibia-up, Tibia-midup, Tibia-midlow and Tibia-low from the proximal to the distal end (Fig. 4). For the knee, the spring elements with extremely low elasticity coefficient were attached to the elements of MCL (medial collateral ligament), ACL (anterior cruciate ligament) and PCL (posterior cruciate ligament) for measuring knee ligament elongation (Fig. 4). The global strain of LCL (lateral collateral ligament) was not considered since LCL tends to loose or slightly tension in the impact simulations. Comparisons of predicted injury risk metrics between the isolated lower limb models and full body model were conducted to evaluate the predicting capability of different isolated lower limb models.
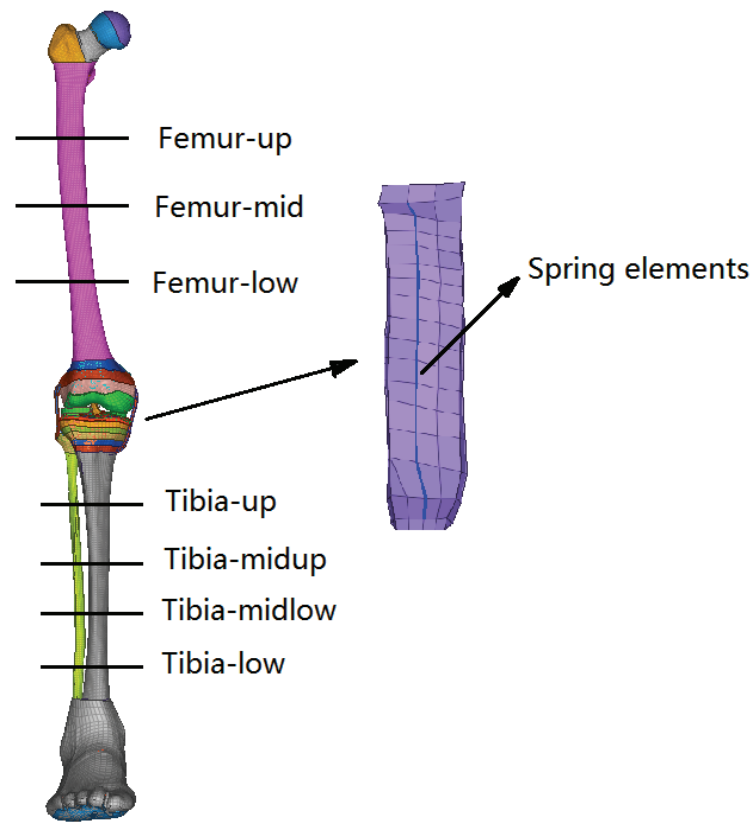

Fig. 4. Knee ligament elongation approach and leg bending moment measuring point

\section{Results}

\subsection{Struck leg kinematics}

Dynamic response of partial simulation models is shown in Figs. 5 and 6 (Full refers the full body model, mass $=0 \mathrm{~kg}$ refers the isolated lower limb model without buttock, $m=2 \mathrm{~kg}, m=4 \mathrm{~kg}$ and $m=7 \mathrm{~kg}$ refer the isolated models with attached buttock mass of $2 \mathrm{~kg}, 4 \mathrm{~kg}$ and $7 \mathrm{~kg}$, respectively) for Shape 1 (low bumper and BLE) and Shape 2 (high bumper and BLE), respectively. It can be seen based on the kinematic response that the lower limbs of different models have no significant difference within $20 \mathrm{~ms}$ in the simulation. However, the isolated lower limbs gradually show difference in kinematics from the full body model after $20 \mathrm{~ms}$, and a significant difference can be observed when it reached $50 \mathrm{~ms}$. This difference is more obvious for the isolated lower limb model without buttock and those with a attached buttock mass of $4 \mathrm{~kg}$ and above. It can be seen that the upper body mass plays an important role in the kinematic response of the lower limbs. In particular, the isolated lower limb model without buttock predicts less knee bending than the full body model. The isolated lower limb models with a attached buttock mass of $4 \mathrm{~kg}$ and above predict over bending to the knee and continuance longer than the full body model.
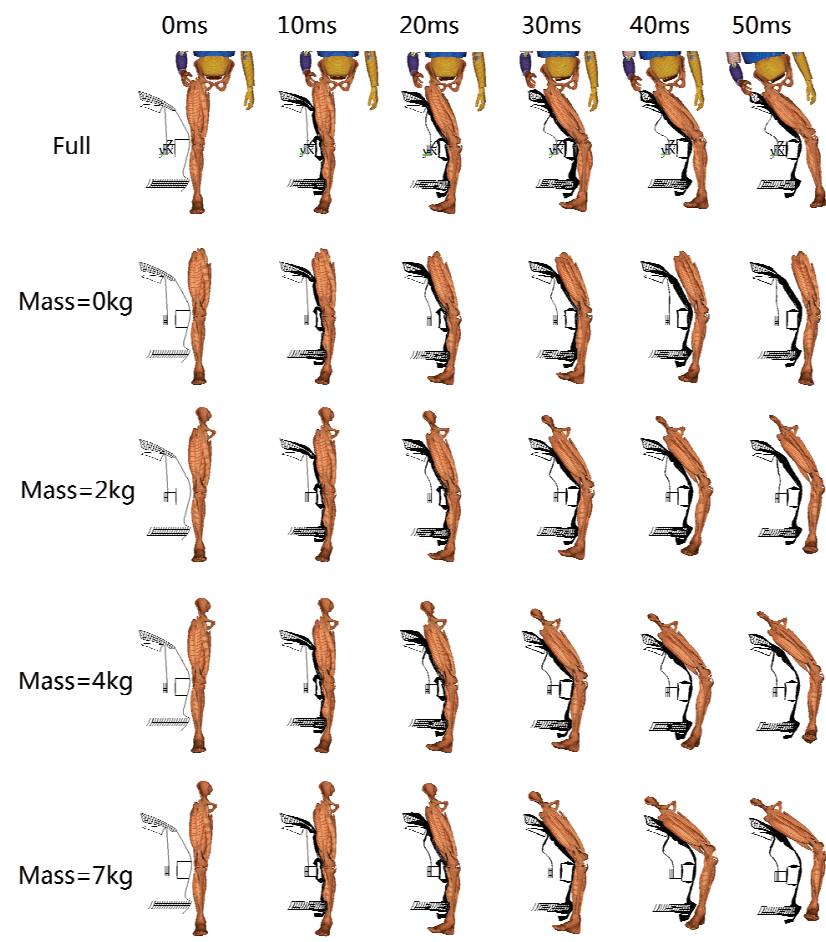

Fig. 5. Predicted pedestrian lower limb kinematics (the struck leg) in Shape 1 collisions 


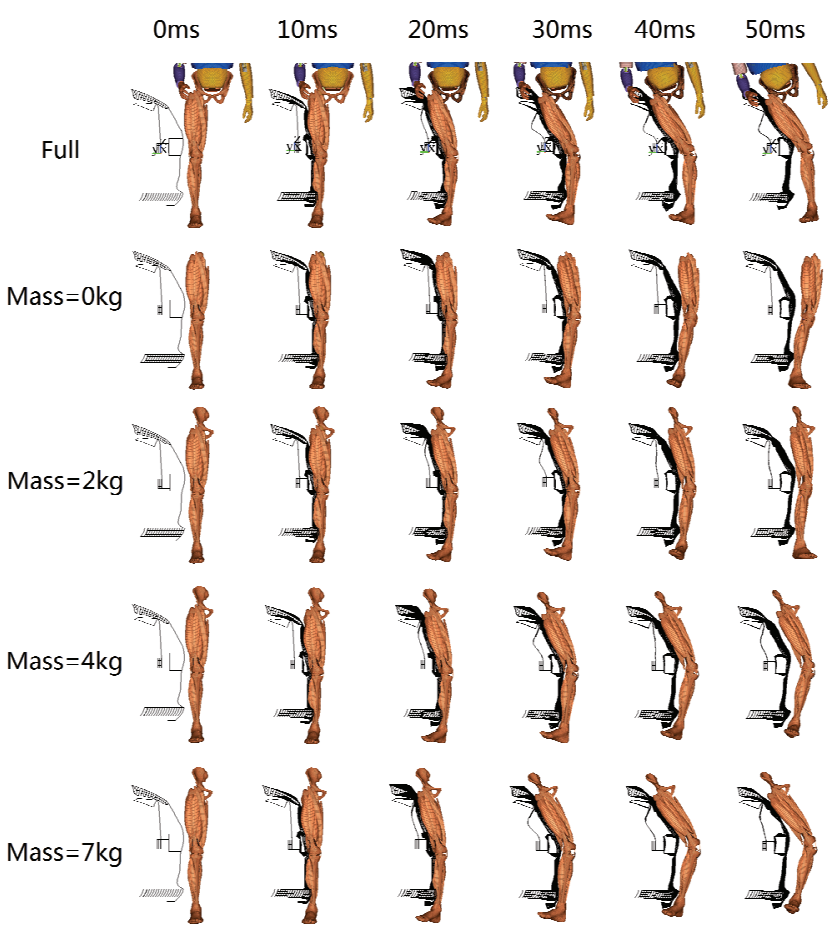

Fig. 6. Predicted pedestrian lower limb kinematics (the struck leg) in Shape 2 collisions

\subsection{Injury measuring}

In Figs. 7 and 8, compared are the time history curves of femur bending moment, knee ligament elongation and tibia bending moment between partial isolated lower limb models $(m=0 \mathrm{~kg}, m=2 \mathrm{~kg}, m=4 \mathrm{~kg}$ and $m=7 \mathrm{~kg}$ ) and the full body model for Shape 1 and Shape 2 impacts, respectively. Obvious difference in the predictions between the isolated lower limb models and full body model can be observed from these curves, especially for femur and knee. Tables A1 and A2 show the peak value of the predicted injury metrics for the full body model and isolated lower limb models, which were extracted from the time history curves of the injury metrics (see the Appendix). It should be noted that the damping phase in the knee ligament elongation curves was not considered in the comparison since the damping is from the spring element which were defined for measuring knee ligament elongation, but not the knee ligaments themselves. Thus, the peak value for knee ligament elongation was extracted at the time of rapture occurring or maximum value (ligament not rapture).
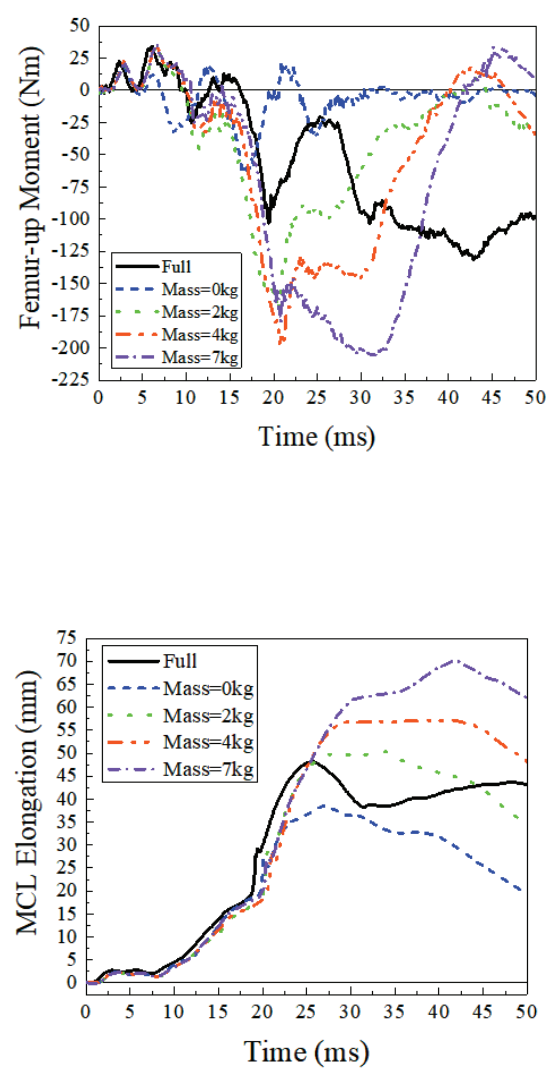

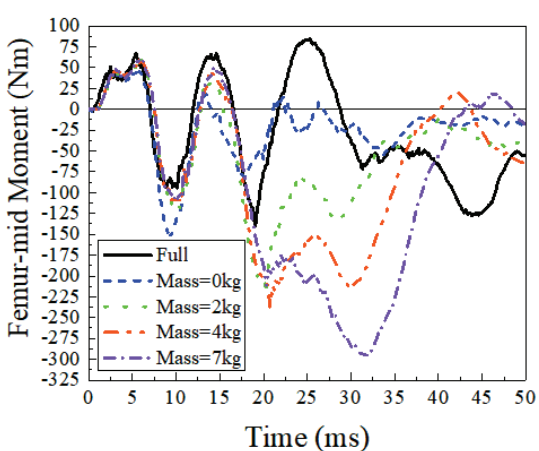

(a) Femur bending moment

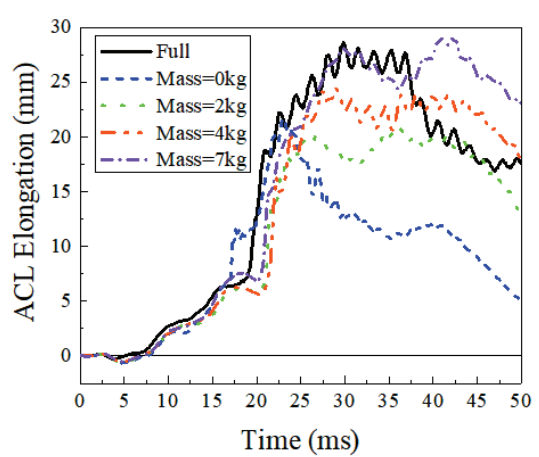

(b) Knee ligament elongation
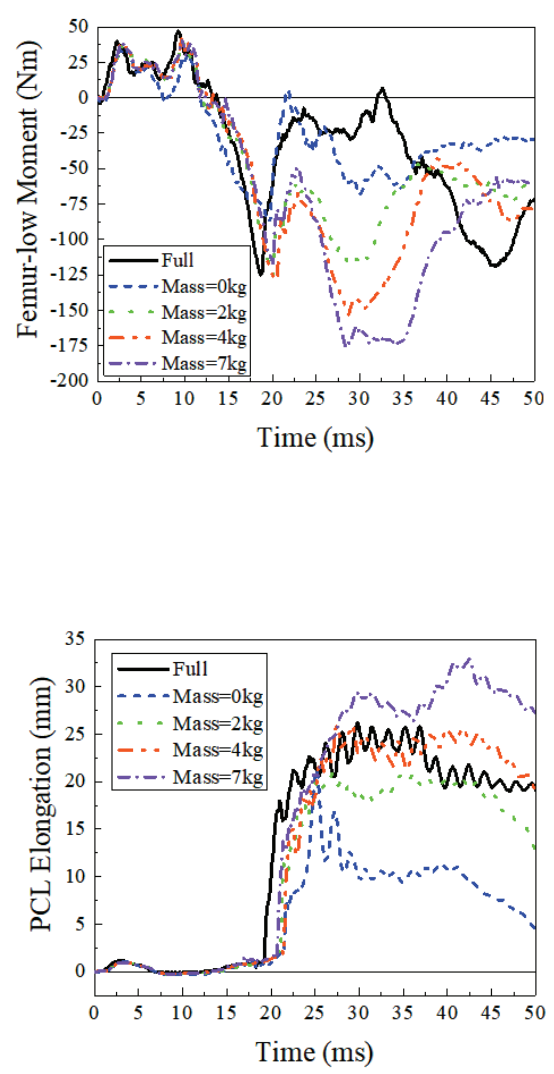

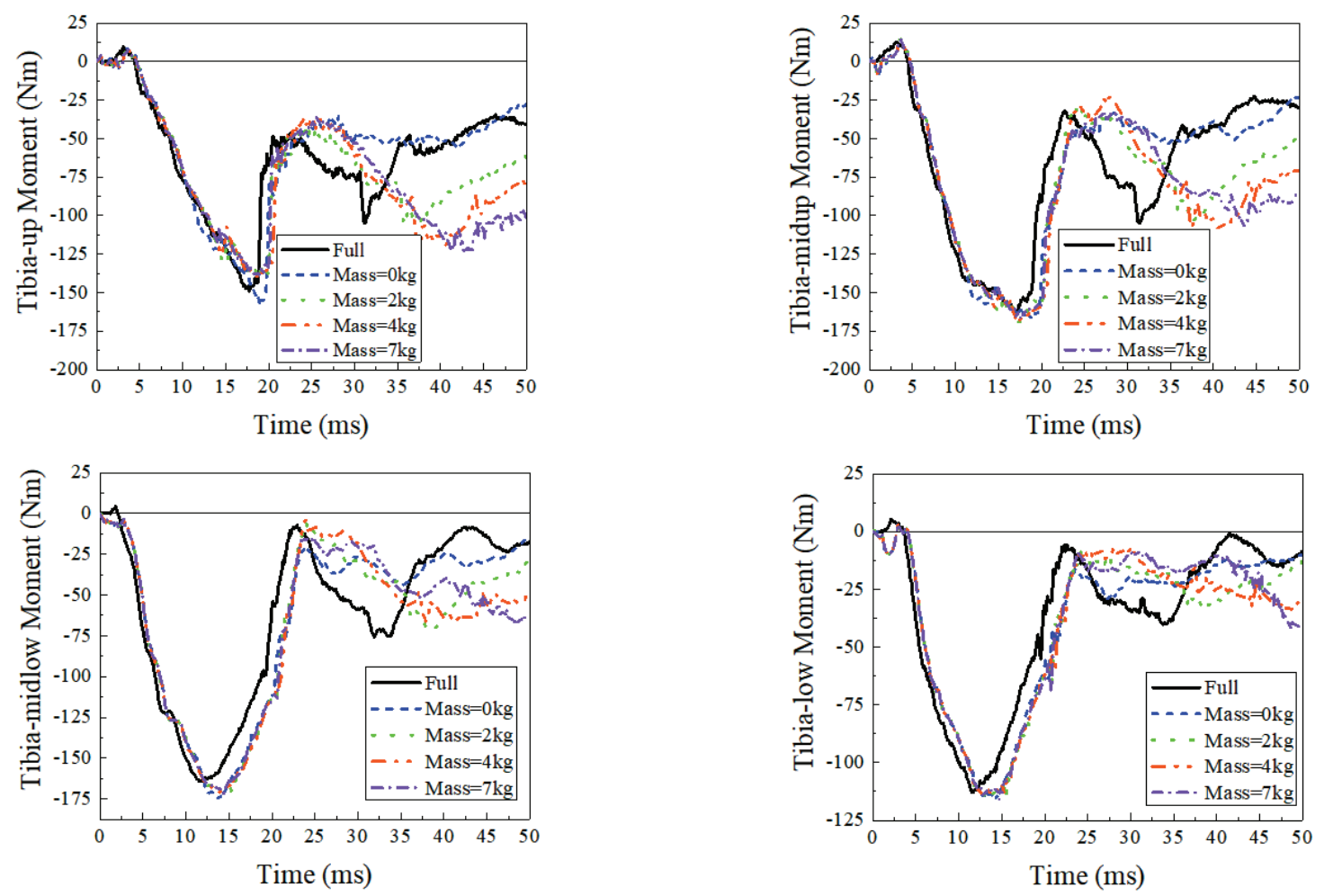

\section{(c) Tibia bending moment}

Fig. 7. Predicted pedestrian lower limb injuries time history (the struck leg) in Shape 1 collisions, comparing the data of full body model and isolated lower limb models with different buttock weight attached
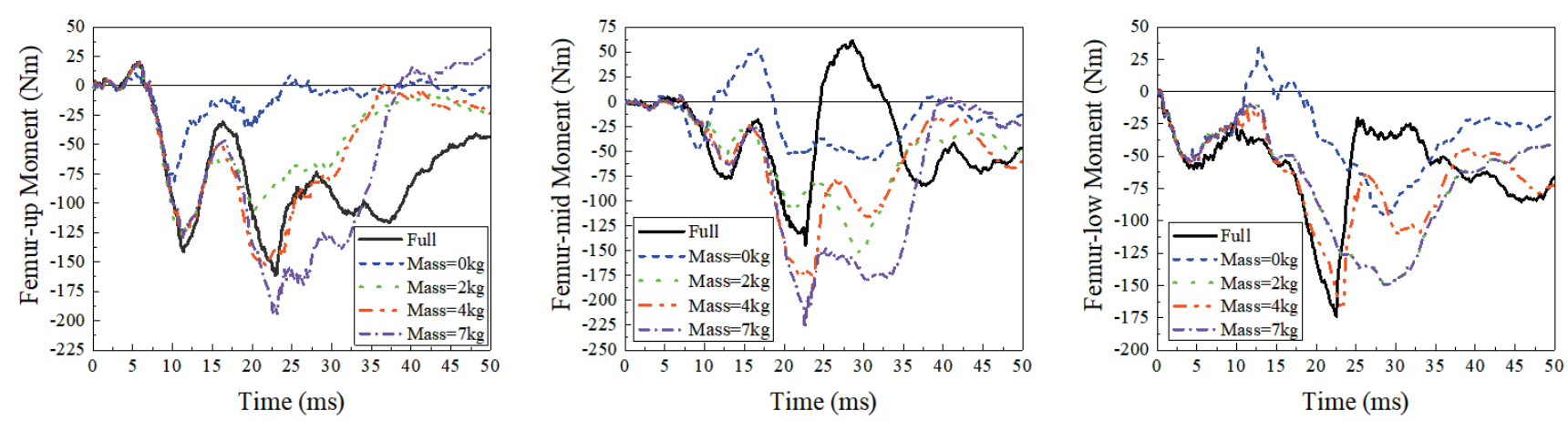

(a) Femur bending moment
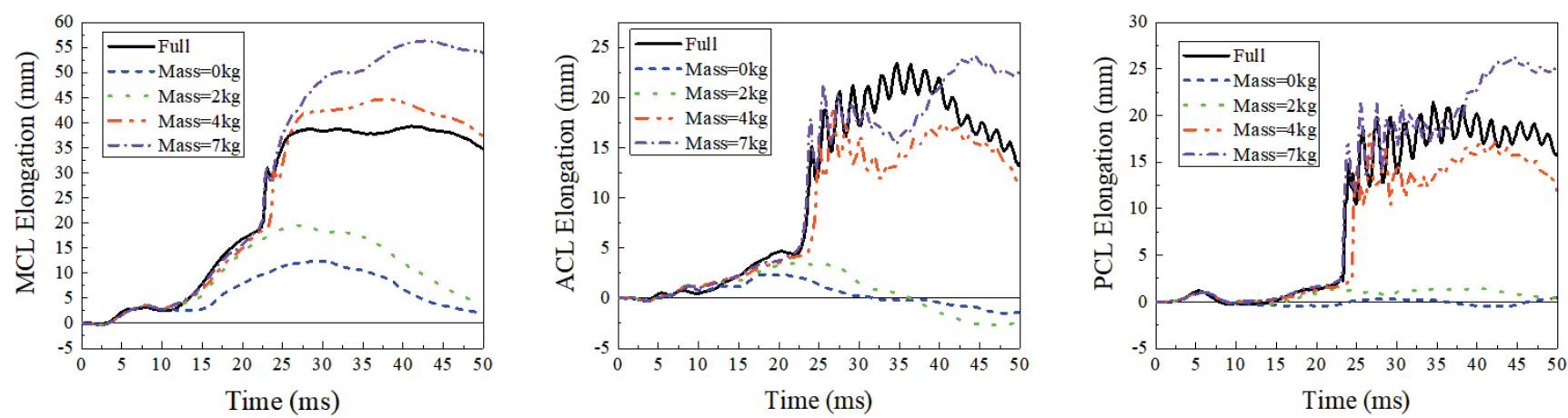

(b) Knee ligament elongation 

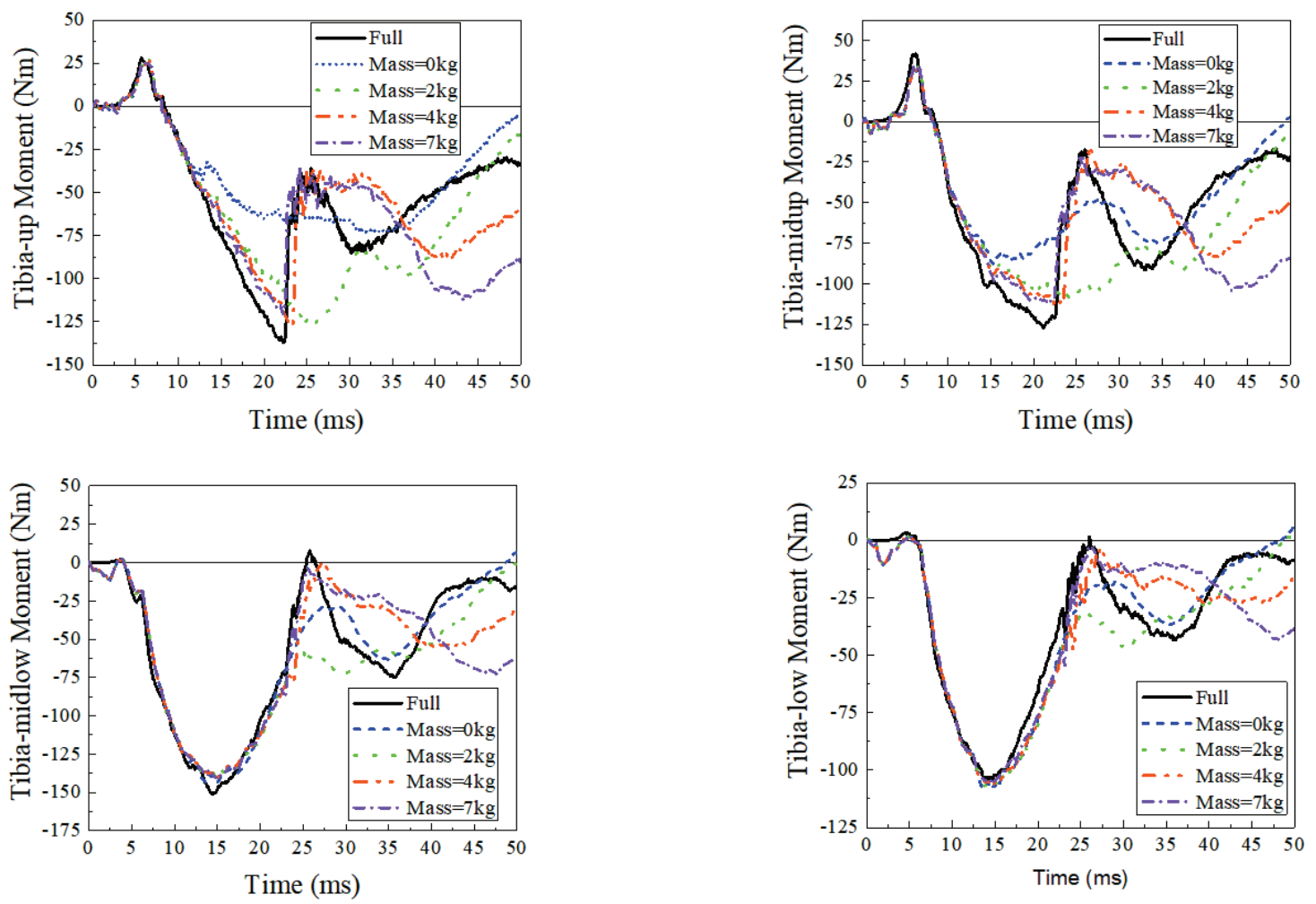

(c) Tibia bending moment

Fig. 8. Predicted pedestrian lower limb injuries time history (the struck leg) in Shape 2 collisions, comparing the data of full body model and isolated lower limb models with different buttock weight attached
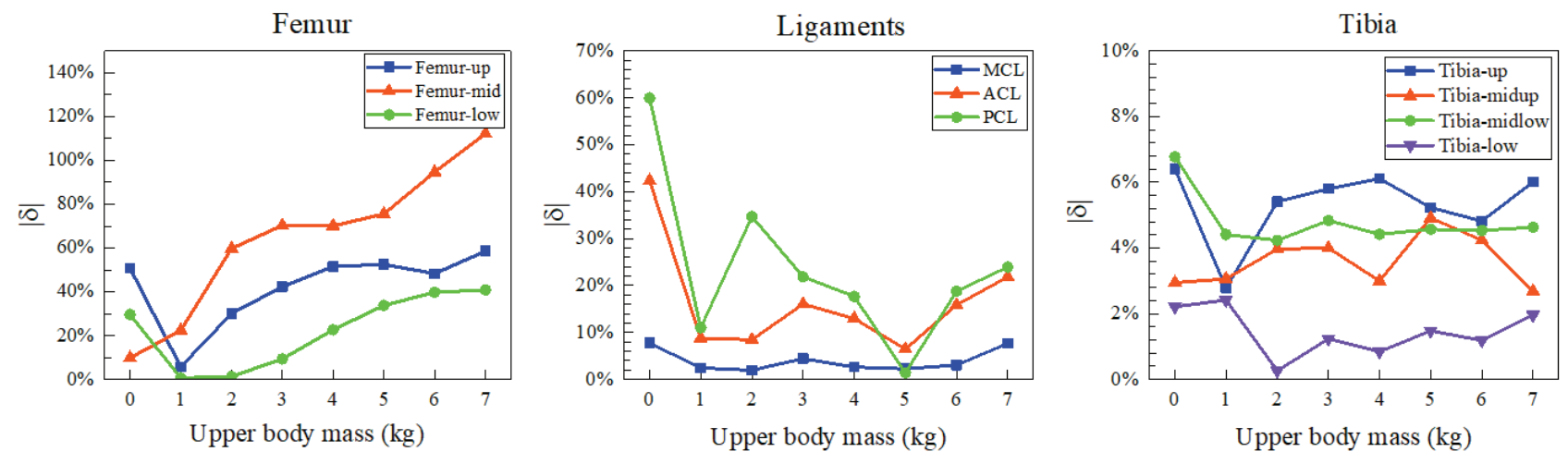

Fig. 9. Absolute value of the relative error for lower limb injury parameters (the struck leg) in Shape 1 collisions
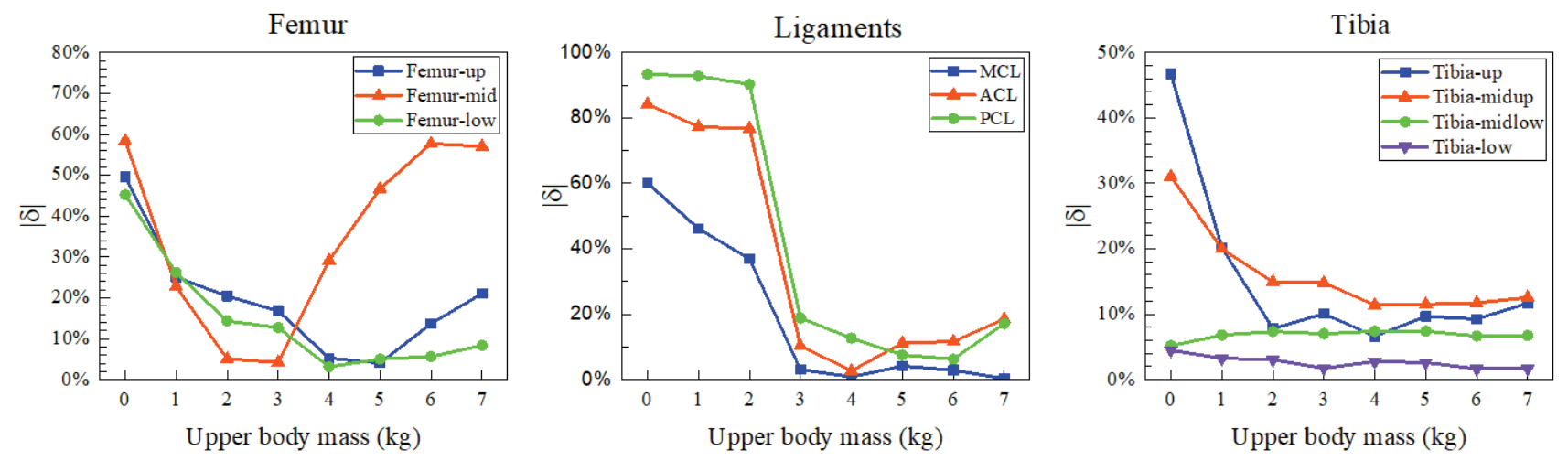

Fig. 10. Absolute value of the relative error for lower limb injury parameters (the struck leg) in Shape 2 collisions 
To assess the difference between predictions from isolated lower limb models and the full body model, the absolute value of the relative error for each injury metric was calculated based on the data shown in Tables 1 and 2 by Eq. (1). Figures 9 and 10 show the absolute relative errors as a function of attached buttock mass and injury metric for Shape 1 and Shape 2 impacts, respectively. Clearly, the upper body mass (the attached buttock) has a significant effect on prediction capability of the isolated lower limb models.

$$
|\delta|=\left|\frac{\text { Peak }_{\text {Isolated }}-\text { Peak }}{\text { Full }}\right| \times 100 \% .
$$

\section{Discussion}

\subsection{Biomechanical understanding}

In vehicle-to-pedestrian impacts, the thigh and lower leg loading condition were depicted by Figs. 11 and 12 , respectively. In Figure 11, $F_{\text {mass }}$ is the inertia force from the upper body mass, $F_{\text {vehicle }}$ is the impact force from the vehicle front end, $F_{\text {lower }}$ is the inertia force from the lower leg and $M_{\text {lower }}$ is the bending moment from the knee. Similarly, in Figure 12, $F_{\text {thigh }}$ is the inertia force from the thigh, $F_{\text {vehicle }}$ is the impact

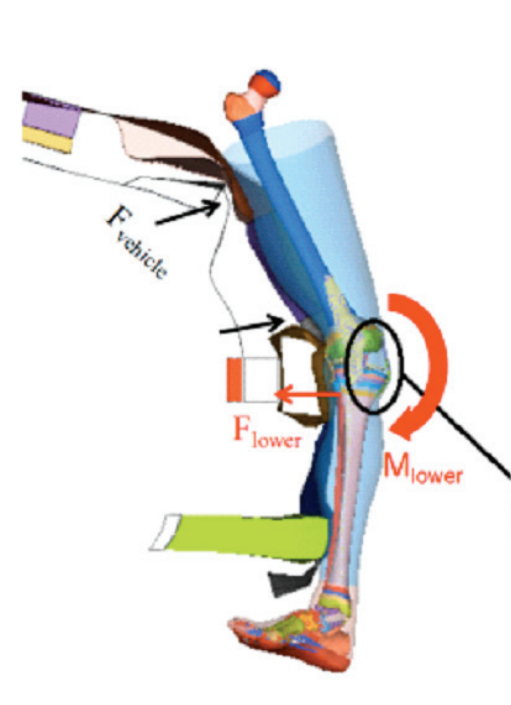

(a)

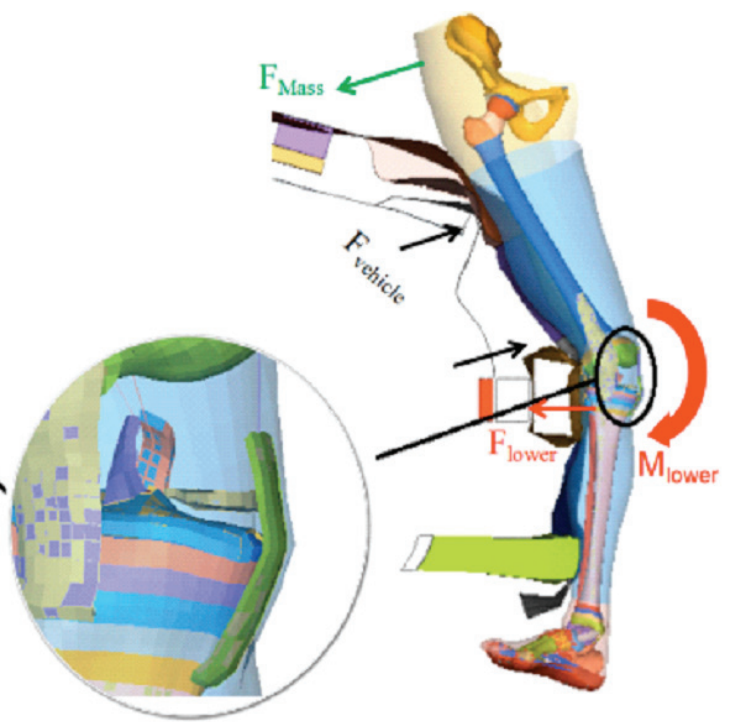

(b)

Fig. 11. Femur loading of (a) $m=0 \mathrm{~kg}$ and (b) $m=1 \mathrm{~kg}$ in Shape 1 impact

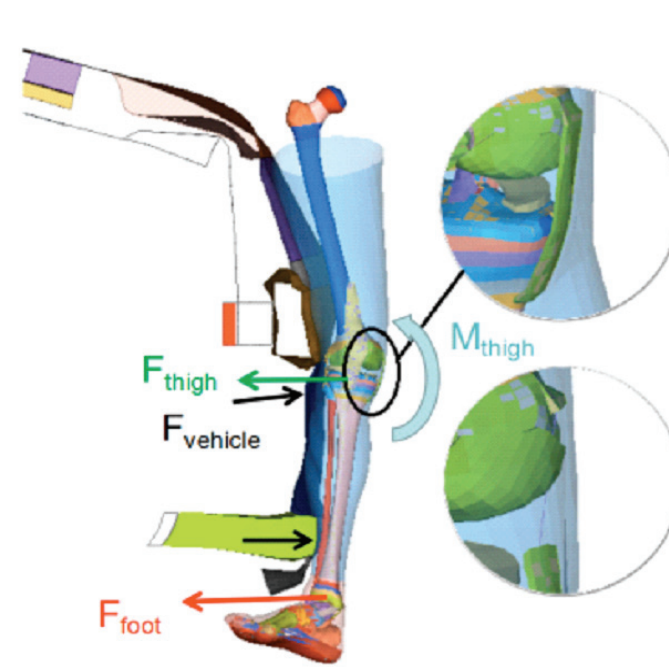

(a)

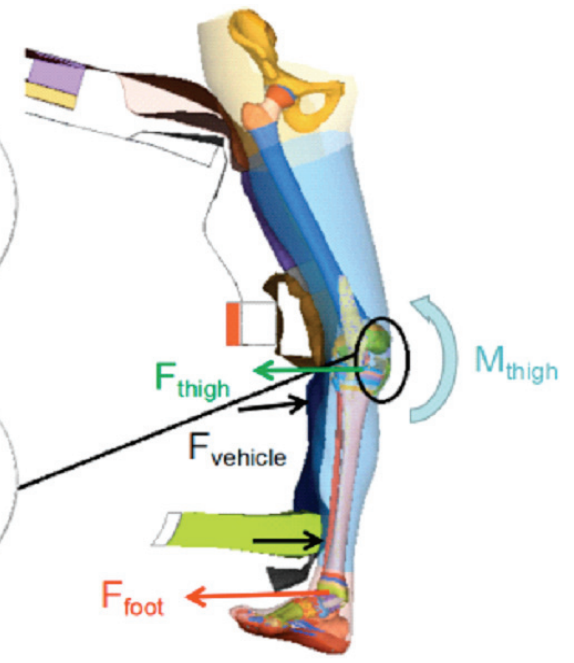

(b)

Fig. 12. Tibia loading of (a) $m=0 \mathrm{~kg}$ and (b) $m=3 \mathrm{~kg}$ in Shape 2 impact 
force from the vehicle front end, $F_{\text {foot }}$ is the inertia force from the foot and $M_{\text {thigh }}$ is the bending moment from the knee.

It can be clearly seen from Figs. 11 and 12 that when $m=0$ the isolated lower limb without buttock cannot bend as that with upper body mass weight attached during the impact. This phenomenon is more pronounced in Shape 2 impacts for the relatively higher bonnet leading edge. This is due to the fact that when no buttock or a light buttock was attached, the lack or inadequacy of $F_{\text {mass }}$ action causes lower bending moment at the proximal and distal of the femur. Thus, the predicted femur bending moment generally increases with increasing the mass of buttock (Tables A1, A2). Compared to the full body model in Shape 1 impacts, the predicted femur bending moments from the isolated lower limb model have a high relative error for the cases of without buttock and with buttock mass larger than $1 \mathrm{~kg}$ (Fig. 9), where the former is lack of upper body mass and the latter is over attached of mass (Table A1). When $m=1 \mathrm{~kg}$, the predicted femur bending moments from the isolated lower limb model are generally close to the full body case, with the relative errors lower than $22 \%$ (Fig. 9). Similar trend was observed for Shape 2 impacts, but good predictions (relative error $<20 \%$ ) of the isolated lower limb model are to the cases of $m>2 \mathrm{~kg}$ for the proximal and distal femur bending moment and $m=2-3 \mathrm{~kg}$ for the mid-shaft femur (Fig. 10). These differences between Shape 1 and Shape 2 impacts are largely due to the shape effect [12], [25]. These findings may suggest that the upper body mass has a significant influence on pedestrian femur injury risk and the effect varies based on vehicle front-end shape.

For knee ligaments, in the Shape 1 collisions, although the ligament is broken like the full body when the buttock mass is not considered ( $m=0 \mathrm{~kg}$, Fig. 11), the first peak after the break is obviously small (Table A1), thus the relative error is still large (Fig. 9). When the attached buttock mass reaches $1 \mathrm{~kg}$ and above, the predicted knee ligament elongation values from the isolated lower limb models are basically similar to the full body model. In the Shape 2 case, when the buttock mass is less than $3 \mathrm{~kg}$, the knee ligaments isolated lower limb model did not tear in the simulation, so the measured ligament elongation values are obviously smaller than the full body model (Table A2), and larger errors are obtained (Fig. 10). In contrast, when the buttock mass reaches $3 \mathrm{~kg}$ and beyond, the ligaments in the isolated lower limb models ruptured and the measured knee ligament elongation values show good agreement with the full body model.
The above observation is mainly from the biomechanical mechanism of knee ligament injuries, where lack of $F_{\text {mass }}$ leads lower bending to the knee and hence less tension to the ligaments [24]. The relative error data in Figs. 9 and 10 show that the isolated lower limb models with buttock mass of above $1 \mathrm{~kg}$ and $3 \mathrm{~kg}$ generally have good predicting capability of knee injury risk, compared to the full body model for Shape 1 and Shape 2, respectively. These results indicate that the upper body mass also has a significant influence on knee injury risk and the effect is not remarkable when the attached upper body mass reaches a certain value, which is determined by vehicle frontend shape, since the relative height between bumper and knee is a crucial factor for knee injury risk [12].

The tibia loading condition shown in Fig. 12 indicates that the effect of upper body mass on tibia injury risk is not direct since the effect would transfer through knee ligaments (especially the MCL). Combining tibia bending moment values from Figs. 7 and 8 , it was found that Tibia-up and Tibia-midup measurements maintain a high correlation with MCL deformation, while Tibia-midlow and Tibia-low measurements do not significantly change with changes in upper body mass. For Tibia-up and Tibia-midup, the bending moment comes from the ligaments tension of the knee joint, and the peak value generally appears at the time when the maximum elongation before MCL rupture and is also the time when the MCL tension is maximum. When the MCL breaks, its tension disappears instantaneously, and the peak value of the bending moments of Tibia-up and Tibia-midup drop suddenly (Fig. 7 at $20 \mathrm{~ms}$ and Fig. 8 at about $22.5 \mathrm{~ms}$ ). Thus, in the Shape 1, the upper body mass has no obvious effect on the proximal tibia injury risk. As predicted, tibia bending moments from all isolated lower limb models show good agreement with the full body condition (Fig. 9), since MCL rupture occurred in all simulations and the MCL produced similar maximum tension before fracture, which leads to the approximative maximum measured peak bending moment values of tibia proximal. However, for the Shape 2 case, when the attached buttock mass is less than $2 \mathrm{~kg}$, the MCL is not broken, which means the proximal tibia is subjected to a small ligament tension, resulting in a smaller proximal tibia bending moment (Table A2). The predicted relative errors of bending moment at proximal and upper shaft tibia in these cases are larger (more than $20 \%$, Fig. 10). When the mass of the upper body reaches $2 \mathrm{~kg}$, although the MCL was also not broken, the MCL deformation almost reached the MCL failure strain at about $22.5 \mathrm{~ms}$ (Fig. $8 \mathrm{MCL}$ elongation), so this produces a suffi- 
ciently large tension to cause a similar proximal tibia bending moment value (Fig. 8 Tibia bending moment), resulting in a smaller relative error $(<20 \%)$ (Fig. 10). Furthermore, when the attached buttock mass reaches $3 \mathrm{~kg}$, the relative errors in tibia bending moment are stable within 20\% (Fig. 10). Distal tibia injury is mainly determined by the lower extremity mass below the lower bumper stiffener [16], so the Tibia-midlow and Tibia-low bending moments change little with changing the upper body mass (Fig. 10). These findings suggest that the effect of upper body mass on pedestrian lower leg injury risk is not as significant as that for the femur and knee, especially in impacts with vehicles having a relatively lower bonnet leading edge height which causes larger bending in the knee, hence, leading to earlier ligaments rupture.

\subsection{Further analysis}

The above findings suggest that a higher buttock mass is needed for vehicle front-end with a higher bumper and bonnet leading edge height. Therefore, in order to explore in which ratio the buttock mass should be changed for differing frontal geometries according to the used Chinese 50th percentile adult male lower limb model. Simulations using another two vehicle front-ends of different BLE and bumper heights, named as Shape 0 and Shape 3 (Fig. 13), were carried out similarly to previous modeling of Shape 1 and Shape 2. Since the BLE height (BLEH) of Shape 0 $(609 \mathrm{~mm})$ was lower than that of Shape $1(664 \mathrm{~mm})$, only simulations with buttock mass of 0 and $0.5 \mathrm{~kg}$ were considered. Similarly, the simulations for Shape 3

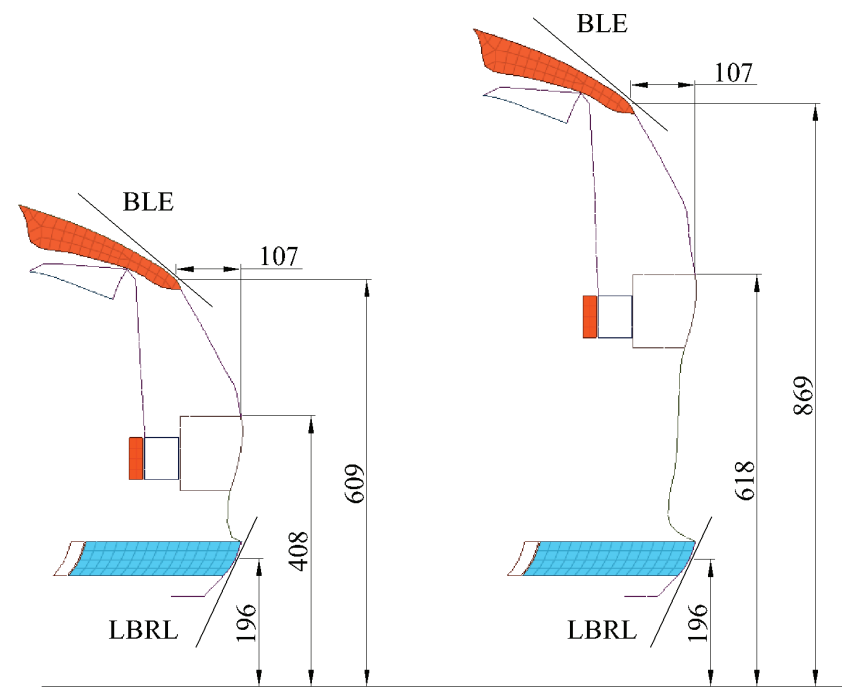

(a)

(b)

Fig. 13. Vehicle front-end buck models: (a) Shape 0 and (b) Shape 3
$($ BLEH $=869 \mathrm{~mm}$ ) focus on buttock mass above $3 \mathrm{~kg}$ considering the optimal buttock mass of $3 \mathrm{~kg}$ for the Shape $2(B L E H=754 \mathrm{~mm})$.

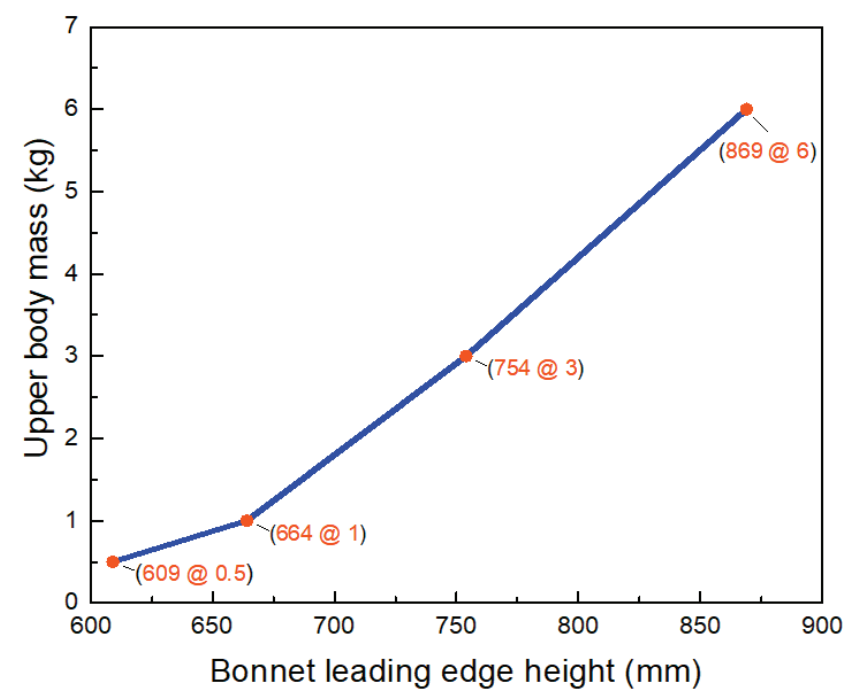

Fig. 14. Optimal buttock mass as a function of BLEH

The simulation results show that the optimal buttock mass for Shape 0 and Shape 3 is $0.5 \mathrm{~kg}$ and $6 \mathrm{~kg}$ (predicted relative errors of injury parameters within $20 \%$ ), respectively. In Figure 14, the optimal buttock mass as a function of BLEH is shown. It can be seen that the higher the BLE is, the larger the upper body mass is required for the isolated lower limb, i.e., there is a positive correlation trend between upper body mass and BLE height. This phenomenon is well explained by the fact that for lower BLE vehicles, the collision position of pedestrian lower limb is not as high as that of higher BLE vehicles, where the collision point is far away from the body's center of mass. Thus, in collisions with lower BLE vehicles, pedestrians are more likely to turn over relative to the vehicle, and the effect of the upper body inertia force is not obvious. For higher BLE vehicles, where the collision position is closer to the center of mass, more velocity is transferred to pedestrians, which changes in extreme case, but naturally, the kinematics from somersault into clear parabolic motion, hence, larger force will transfer to the pedestrians. However, in the collisions the front of the vehicle first contacts with the lower limbs, the upper body will inevitably produce a huge inertial reaction force on the lower limbs to prevent its movement. Therefore, in the isolation study, for the high BLE vehicles, a larger upper body mass is needed to ensure the same leg biomechanical response as the whole body condition. In addition, in the process of looking for a suitable upper body mass of the isolated lower limb for Shape 3, it was found 
that when the upper body load reaches or exceeds $6 \mathrm{~kg}$, the relative errors of the injury measurements change slightly. It is not difficult to introduce that when the BLE is high enough, the buttock of the lower limbs will directly hit the BLE or below in vehicle collisions (Fig. 15). In this case, the buttock that represent the upper body are directly blocked by the front structure of the vehicle. Therefore, a suitable upper body load is a feasible solution of not less than a certain value.

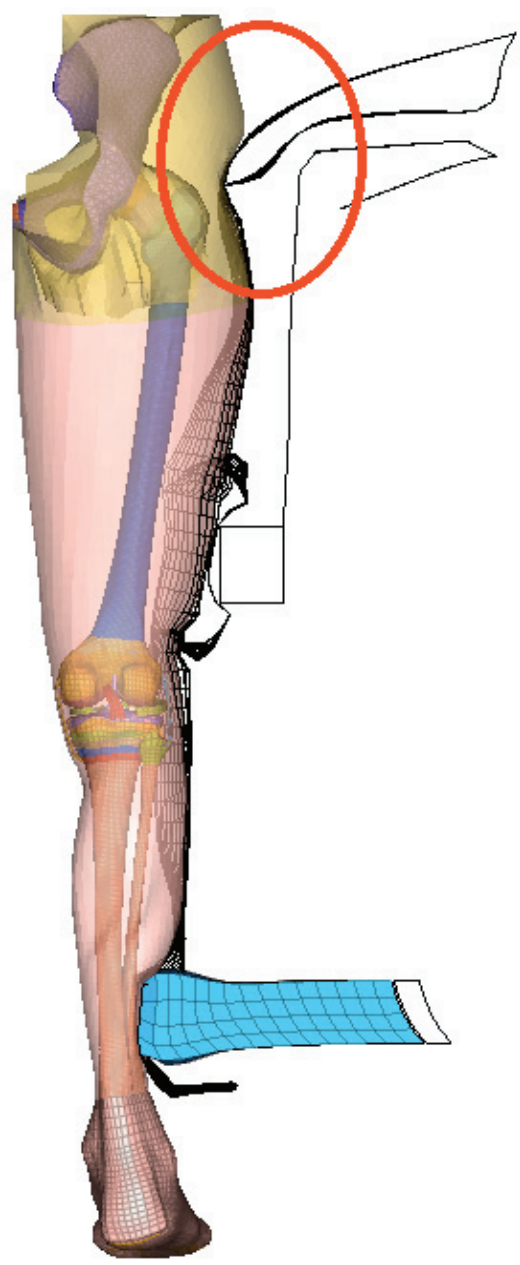

Fig. 15. Kinematics of the isolated lower limb model in collision with Shape 3

\subsection{Limitations}

In the current work, only the limited impact scenarios and isolated conditions were considered, the optimal isolated lower limb configuration for predicting full body biomechanical response in all impact scenarios cannot be determined based on the current results, even though the isolated lower limb models with good predicting capability of Chinese full body model for the studied impact scenarios $(40 \mathrm{~km} / \mathrm{h}$ impacting with four shapes) can be roughly obtained (Fig. 14). Only variations of bumper and BLE height were considered in the current study for vehicle front-end designs. The effect of the horizontal distance between BLE and the actual end of the front-end in the section plane (defined as BLE depth in [12] and [15]) on the selection of buttock mass has not been considered in the current work, which would affect the loading condition on the pedestrian femur and pelvis, hence, influence of upper body mass on lower limb response. The effect of lower bumper stiffener height and depth on the selection of buttock mass also has not been considered. But this might be less of importance than that of BLE and bumper geometry according to the above mechanical analysis, and the variation of lower bumper stiffener depth is not obvious in modern vehicles [15]. The work is to be continued to advance in this respect based on optimization methods considering a broad range of impact scenarios (e.g., different BLE, lower bumper stiffener depths and vehicle front-end stiffness levels) and isolated configurations (e.g., different attaching locations and attaching form of buttock mass).

\section{Conclusions}

In the current study, predictions of pedestrian leg injury metrics from different isolated human body lower limb models were compared to that of a full body pedestrian model in vehicle-to-pedestrian collisions considering different vehicle front-end shapes. The results indicate that upper body mass has a significant influence on pedestrian lower limb injury risk, the effect varies from vehicle bonnet leading edge height and is more remarkable to the femur and knee ligaments than to the tibia. In particular, the upper body mass can generally increase femur and knee ligaments injury risk, but has no obvious effect on the injury risk of tibia. The results also show that a higher attached buttock mass is needed for isolated pedestrian lower limb model for impacts with vehicles of higher bonnet leading edge. The findings of this study may suggest that it is necessary to consider vehicle shape variation in assessment of vehicle pedestrian protection performance and leg-form impactors with adaptive upper body mass should be used for vehicles with different front-end shapes, and the use of regional leg-form impactor modeling the local anthropometry to evaluate the actual lower limb injury of pedestrians in different countries and regions. 


\section{Acknowledgement}

This work was supported by the National Natural Science Foundation of China (Grant No. 51805162), Natural Science Foundation of Hunan Province (Grant No. 2018JJ3532 and 2019JJ40021) and Scientific Research Fund of Hunan Provincial Education Department (Grant No. 18A188).

\section{References}

[1] Beillas P., Begeman P.C., Yang K.H., King A.I., ARnoux P.J., Kang H.S., Prasad P., Lower limb: advanced FE model and new experimental data, SAE Technical Paper No. 2001-22-0022, 2001.

[2] Cardot J., Masson C., Arnoux P.J., Brunet C., Finite element analysis of cyclist lower limb response in car-bicycle accident, Int. J. Crashworthiness, 2006, 11 (2), 115-130.

[3] Chirag S., George H., Mark B., Development of an FE model for FlexPLI with upper body mass for enhanced pedestrian safety assessment, Proceedings of 26th International Conference on the Enhanced Safety of Vehicles (ESV), 2019, Eindhoven, Netherlands, Paper No. 19-0228.

[4] C-NCAP, China New Car Assessment Programme Management Regulation (2018 version), China Automotive Technology and Research Center, 2018.

[5] Euro-NCAP, Pedestrian Testing Protocol, Version 8.4, European New Car Assessment Programme, 2017.

[6] GUAN T., Modeling and application of a China 50th percentile pedestrian lower limb finite element model, Dissertation, Hunan University, 2019.

[7] HUSAF, Chinese Human Dummy, Hunan SAF Automobile Technology Co. Ltd., 2019, https://https://www.hnsaf.com/en/ index.html. Accessed 14 September 2019.

[8] J-NCAP, Car Safety Performance Guidelines, New Car Assessments 2014.3, 2014.

[9] Konosu A., Tanahashi M., Development of a biofidelic flexible pedestrian legform impactor, SAE Technical Paper No. 2003-22-0020, 2003.

[10] Konosu A., TANahashi M., Development of an FE flexible pedestrian leg-form impactor (Flex-PLI 2003R) model and evaluation of its biofidelity, SAE Technical Paper No. 2004-01-1609, 2004.

[11] Kawabe Y., Pal C., OKuyama H., OKabe T., Flex-PLI application to high-bumper vehicles-optimization of supplemental weight, SAE Int. J. Transp. Saf., 2013, 1 (2), 278-285.

[12] Li G., Lyons M., Wang B., Yang J., Otte D., Simms C., The influence of passenger car front shape on pedestrian injury risk observed from German in-depth accident data, Accid. Anal. Prev., 2017, 101, 11-21.

[13] Li G., Ma H., Guan T., Gao G., Predicting safer vehicle font-end shapes for pedestrian lower limb protection via a numerical optimization framework, Int. J. Automot. Technol., 2020, 21 (3), 749-756.

[14] Li G., TAN Z., Lv X., REN L., Numerical reconstruction of injuries in a real world minivan-to-pedestrian collision, Acta Bioeng. Biomech., 2019, 21 (2), 21-30.
[15] Li G., Wang F., Otte D., Cai Z., Simms C., Have pedestrian subsystem tests improved passenger car front shape, Accid Anal. Prev., 2018, 115, 143-150.

[16] Li G., YAng J., Simms C., The influence of gait stance on pedestrian lower limb injury risk, Accid Anal. Prev., 2015, 85, 83-92.

[17] Mo F., Arnoux P.J., Avalle M., Scattina A., Semino E., MASSON C., Incidences of various passenger vehicle frontend designs on pedestrian lower limb injuries, Int. J. Crashworthiness, 2015, 20(4), 337-347.

[18] Mo F., Li F., Behr M., Xiao Z., Zhang G., Du X., A lower limb-pelvis finite element model with $3 D$ active muscles, Ann. Biomed. Eng., 2018, 46 (1), 86-96.

[19] Mo F., Li J., Dan M., LiU T., Behr M., Implementation of controlling strategy in a biomechanical lower limb model with active muscles for coupling multibody dynamics and finite element analysis, J. Biomech., 2019, 91, 51-60.

[20] Mo F, Li J, YANG Z, ZHOU S, BEHR M. In vivo measurement of plantar tissue characteristics and its indication for foot modeling, Ann. Biomed. Eng., 2019, 47 (12), 2356-2371.

[21] Mao H., Zhang L., Jiang B., GenthiKatti V.V., Jin X., ZHU F., YANG K.H., Development of a finite element human head model partially validated with thirty five experimental cases, J. Biomech. Eng., 2013, 135 (11), 111002-111015.

[22] Mizuno Y., Summary of IHRA pedestrian safety working group activities - Proposed test methods to evaluate pedestrian protection offered by passenger cars, Proceedings of the 19th International Technical Conference of Enhanced Safety of Vehicles (ESV), 2005, Washington, USA, Paper No. 05-0138-O.

[23] NIE B., ZHOU Q., Can new passenger cars reduce pedestrian lower extremity injury? A review of geometrical changes of front-end design before and after regulatory efforts, Traffic Inj. Prev., 2016, 17(7), 712-719.

[24] Petit P., Trosseille X., Dufaure N., Dubois D., Potier P., VALlanciEn G., The effect of upper body mass and initial knee flexion on the injury outcome of post mortem human subject pedestrian isolated legs, SAE Technical Paper No. 2014-22-0008, 2014.

[25] Scattina A., Mo F., Masson C., Avalle M., Arnoux P. J., Analysis of the influence of passenger vehicles front-end design on pedestrian lower extremity injuries by means of the LLMS model, Traffic Inj. Prev., 2018, 19(5), 535-541.

[26] Simms C., Wood D., Pedestrian and Cyclist Impact - A Biomechanical Perspective, Springer, 2009.

[27] Toyota Motor Corporation, Documentation: Total Human Model for Safety (THUMS) AM50 pedestrian/occupant model academic version 4.02 20150527, 2015.

[28] Untaroiu C.D., Shin J., Crandall J.R., Fredriksson R., Bostrom O., TAKahashi Y., KIKUChI Y., Development and validation of pedestrian sedan bucks using finite-element simulations: a numerical investigation of the influence of vehicle automatic braking on the kinematics of the pedestrian involved in vehicle collisions, Int. J. Crashworthiness, 2010, 15 (5), 491-503.

[29] World Health Organization, Global status report on road safety 2018: summary, Geneva, Switzerland, 2018.

[30] Wu T., Kim T., Bollapragada V., Poulard D., Chen H., PANZER M.B., PIPKORN B., Evaluation of biofidelity of THUMS pedestrian model under a whole-body impact conditions with a generic sedan buck, Traffic Inj. Prev., 2017, 18 (Suppl.), S148-S154. 


\section{Appendix}

Table A1. Peak values of injury metrics for different models impacting with the Shape 1

\begin{tabular}{|l|c|c|c|c|c|c|c|c|c|}
\hline \multicolumn{1}{|c|}{ Peak value } & Full & $m=0 \mathrm{~kg}$ & $m=1 \mathrm{~kg}$ & $m=2 \mathrm{~kg}$ & $m=3 \mathrm{~kg}$ & $m=4 \mathrm{~kg}$ & $m=5 \mathrm{~kg}$ & $m=6 \mathrm{~kg}$ & $m=7 \mathrm{~kg}$ \\
\hline Measuring point & & & & & & & & & \\
\hline Femur-up moment [Nm] & 131.01 & 64.38 & 123.52 & 170.48 & 186.45 & 198.58 & 199.81 & 194.28 & 207.76 \\
\hline Femur-mid moment [Nm] & 139.51 & 153.18 & 170.84 & 222.95 & 237.55 & 237.36 & 244.89 & 271.63 & 295.99 \\
\hline Femur-low moment [Nm] & 124.68 & 87.77 & 123.82 & 126.40 & 136.36 & 152.94 & 166.79 & 174.33 & 175.60 \\
\hline MCL elongation [mm] & 29.11 & 26.86 & 28.40 & 28.54 & 27.82 & 28.34 & 28.45 & 28.21 & 26.88 \\
\hline ACL elongation [mm] & 18.75 & 10.81 & 17.11 & 17.17 & 15.74 & 16.32 & 17.54 & 15.78 & 14.66 \\
\hline PCL elongation [mm] & 17.99 & 7.20 & 16.01 & 11.75 & 14.06 & 14.81 & 17.74 & 14.61 & 13.68 \\
\hline Tibia-up moment [Nm] & 148.99 & 158.52 & 144.83 & 140.93 & 140.34 & 139.88 & 141.19 & 141.81 & 140.03 \\
\hline Tibia-midup moment [Nm] & 162.68 & 167.47 & 167.65 & 169.14 & 169.19 & 167.56 & 170.67 & 169.58 & 167.04 \\
\hline Tibia-midlow moment [Nm] & 164.05 & 175.16 & 171.29 & 171.00 & 171.98 & 171.29 & 171.55 & 171.49 & 171.64 \\
\hline Tibia-low moment [Nm] & 113.31 & 115.82 & 116.05 & 113.61 & 114.71 & 114.27 & 114.99 & 114.66 & 115.54 \\
\hline
\end{tabular}

Table A2. Peak values of injury metrics for different models impacting with the Shape 2

\begin{tabular}{|l|c|c|c|c|c|c|c|c|c|}
\hline \multicolumn{1}{|c|}{ Peak value } & Full & $m=0 \mathrm{~kg}$ & $m=1 \mathrm{~kg}$ & $m=2 \mathrm{~kg}$ & $m=3 \mathrm{~kg}$ & $m=4 \mathrm{~kg}$ & $m=5 \mathrm{~kg}$ & $m=6 \mathrm{~kg}$ & $m=7 \mathrm{~kg}$ \\
\hline Feasuring point & & & & & & & & & \\
\hline Femur-up moment $[\mathrm{Nm}]$ & 161.85 & 81.61 & 121.30 & 128.77 & 134.66 & 153.32 & 168.43 & 184.01 & 195.92 \\
\hline Femur-mid moment [Nm] & 145.19 & 60.38 & 112.14 & 152.55 & 151.35 & 187.56 & 212.97 & 228.99 & 227.98 \\
\hline Femur-low moment [Nm] & 174.16 & 95.40 & 128.57 & 149.17 & 152.00 & 168.65 & 165.37 & 164.35 & 159.59 \\
\hline MCL elongation [mm] & 31.01 & 12.37 & 16.70 & 19.55 & 31.97 & 31.28 & 32.30 & 31.92 & 31.11 \\
\hline ACL elongation [mm] & 15.12 & 2.39 & 3.44 & 3.52 & 13.56 & 15.53 & 16.81 & 16.89 & 17.91 \\
\hline PCL elongation [mm] & 14.78 & 0.99 & 1.07 & 1.44 & 11.99 & 12.90 & 15.89 & 15.71 & 17.31 \\
\hline Tibia-up moment [Nm] & 137.29 & 73.15 & 109.57 & 126.58 & 123.46 & 128.17 & 124.04 & 124.57 & 121.24 \\
\hline Tibia-midup moment [Nm] & 127.45 & 87.90 & 101.98 & 108.41 & 108.58 & 112.92 & 112.77 & 112.49 & 111.45 \\
\hline Tibia-midlow moment [Nm] & 151.23 & 143.42 & 140.92 & 140.10 & 140.61 & 140.04 & 140.03 & 141.16 & 141.05 \\
\hline Tibia-low moment [Nm] & 103.79 & 108.45 & 107.17 & 106.96 & 105.60 & 106.66 & 106.50 & 105.51 & 105.52 \\
\hline
\end{tabular}

